\title{
Allele Specific and Locus Non-specific Suppressors in Aspergillus nidulans
}

\author{
By JERZY BAL, IWONA E. KOWALSKA, DOROTA M. MACIEJKO \\ AND PIOTR WĘGLEŃSKI \\ Department of Genetics, Warsaw University, Al. Ujazdowskie 4, \\ 00-478 Warsaw, Poland
}

(Received 15 August 1978; revised 26 April 1979)

\begin{abstract}
Using $N$-methyl- $N^{\prime}$-nitro- $N$-nitrosoguanidine, ultraviolet irradiation, ethyl methanesulphonate or 4-nitroquinoline-1-oxide mutagenesis and an enrichment method for the isolation of auxotrophs, 25 mutants with defects in the adA locus were obtained after screening 41376 colonies. One of these, adA24, did not complement with any of the other adA mutants, had a very high reversion rate and had some other properties which usually characterize strains carrying nonsense mutations. All revertants of adA24 carried dominant suppressor mutations. A group of adA24 suppressors was tested for allele and locus specificity. They were found to suppress only some adA alleles, and at the same time, some mutations in the meth $G, \operatorname{meth} H, \arg B$ and pro $A$ loci. It is proposed that the allele specific and locus nonspecific adenine suppressors are suppressors of nonsense mutations.
\end{abstract}

\section{INTRODUCTION}

Among Eukaryota, suppressors of nonsense mutations have been identified in yeast (Hawthorne \& Mortimer, 1963; Barben, 1966), Neurospora crassa (Seale, 1968) and probably in Podospora anserina (Picard, 1973) and Drosophila melanogaster (Lindsley \& Grell, 1968). In the case of yeast (Sherman et al., 1970; Stewart \& Sherman, 1972; Stewart et al., 1972; Hawthorne \& Leupold, 1974; Randerath et al., 1975; Piper et al., 1976), and to a lesser extent $N$. crassa (Seale et al., 1977), genetical studies on these suppressors were followed by biochemical analysis involving studies on tRNA structure, which made it possible to distinguish between amber, ochre and opal suppressors. This paper describes the selection and genetical analysis of nonsense suppressors in another eukaryotic organism, Aspergillus nidulans. Preliminary data on these suppressors have been presented in a short communication (Bal et al., 1978).

In bacteria and viruses, several different approaches for the direct identification of nonsense suppressors are available (Benzer \& Champe, 1962; Kennedy \& Crowlesmith, 1975; Mindich et al., 1976). In Eukaryota, no such approach exists. The first nonsense suppressors were found in yeast by Hawthorne \& Mortimer (1963) who studied the frequency of simultaneous reversion of several mutations. They demonstrated co-suppression of mutations in different genes by a single suppressor gene.

To increase the chances of isolating nonsense suppressors a method similar to that used by Seale (1968) in N. crassa was applied. This method involved two steps. Firstly, a number of mutants defective in a particular gene were isolated and from them those showing properties expected for nonsense mutations were chosen. Secondly, suppressors obtained for presumed nonsense mutations were characterized.

We decided to look for nonsense mutations in mutants defective at the adA locus. The enzyme specified by the $a d A$ gene is well characterized and strains carrying adA mutations are unable to convert adenylosuccinate (AMPS) to adenosine 5'-monophosphate (AMP) 
(Foley et al., 1965). They are thus blocked in the last step of AMP biosynthesis (Fig. 1) and metabolic suppressors of $a d A$ mutants are unlikely to be obtained. Furthermore, it is known that certain adA mutations show intragenic complementation (Foley et al., 1965). This helps to select potential nonsense mutations which are unlikely to complement other mutations within the same locus. There are good methods for the selection of mutants blocked in the last stages of adenine synthesis and for distinguishing adA mutations from mutations in other adenine loci (Foley et al., 1965; Bal \& Pieniążek, 1979).

\section{METHODS}

Strains. The following strains were obtained from the Fungal Genetics Stock Center: sulA1, galAI, pyroA4, facA303, lacAl, choAl, chaAl (FGSC 465); biAl, adH23, acrA1, wA3, nicB8 (FGSC 239); adG14, proA1, pabaA1, yA2, wA3 (FGSC 72); adF17, yA2, pabaA1, fpaD11 (FGSC 273); yA2, wA2, thiA4, cnxE16, adD3, biAl (FGSC 268); adB1, veA1 (FGSC 217); riboAl, yA2, adE20, methG1, suCadE20, palC4, pabaB22, pyroA4 (FGSC 517); suAladE20, pyroA4, adE20 (FGSC 47). Strains lysB5, nicB8, veA1, wA3, acrA1, adA3; lysB5, nicB8, veA1, wA3, acrA1, adA55; biA1, yA2, acrA1, riboB2, veA1, adA3 and biA1, yA2, acrA1, riboB2, veAl, adA55 were obtained from Dr C. F. Roberts via Dr H. N. Arst. The adA3 and adA55 mutations were described previously as AM3 and AM55 (Foley et al., 1965). Strains methA17, yA2, proA2 and methD10, yA2, pyroA4 were obtained from the Department of Genetics, Polish Academy of Sciences, Warsaw, Poland. Other strains used in this work originated from the collection of the Department of Genetics, Warsaw University. Mutation terminology was as in Clutterbuck (1973).

Chemicals. 4-Nitroquinoline-1-oxide (NQO) was purchased from ICN (Cleveland, Ohio 44128, U.S.A.), $N$-methyl- $N^{\prime}$-nitro- $N$-nitrosoguanidine (MNNG) and ethyl methanesulphonate (EMS) from Koch-Light and adenylosuccinate (AMPS) from Sigma. $N$-Glycosyl-polifungin was obtained from the Department of Drug Technology and Biochemistry of the Gdańsk Institute of Technology, Poland.

Genetic analysis. The minimal medium (MM) and methods of genetic analysis were standard (Pontecorvo et al., 1953). The frequency of reversion was examined as described by Bal et al. (1977). Haploidization was performed as described earlier (Bal et al., 1975), with pabaA1, acrA1, phenA2, lysB5, nicB8, pyroA4, sB3, riboB2, chaAl (FGSC 146) being used as a 'master strain'.

Mutagenesis. The mutations were induced by ultraviolet (u.v.) irradiation (Pontecorvo et al., 1953), or by treatment with EMS (Fink, 1970), NQO (Bal et al., 1977) or MNNG (Chattoo \& Sinha, 1974; Malling \& De Serres, 1970). The polifungin method (Bal et al., 1974) was used to enrich for adenine auxotrophs.

Biochemical analysis. Adenylosuccinate lyase (EC 4.3.2.2; adenylosuccinate AMP-lyase) activity was assayed by a modification of the method described by Foley et al. (1965). The assay mixture contained, in a final volume of $1 \mathrm{ml}: 0.05 \mathrm{M}$-Tris/ $\mathrm{HCl}$ buffer $\mathrm{pH} 8.5,0.08 \mathrm{M}$-AMPS, 30 to $250 \mu \mathrm{g}$ protein and $15 \%(\mathrm{v} / \mathrm{v})$ glycerol. In the control, AMPS was omitted. Assays were done at $37^{\circ} \mathrm{C}$ with a Beckman model 25 spectrophotometer. One unit of activity of the enzyme was defined as $10^{4} \times \Delta A_{280} \min ^{-1}$ (mg protein) ${ }^{-1}$. Protein was estimated by the method of Lowry. The accumulation of adenine biosynthetic pathway metabolites was determined as described by Bal \& Pieniążek (1979).

\section{RESULTS}

\section{Selection of adA mutations}

Mutations in the adA gene were obtained after EMS, MNNG or NQO treatment. They were induced in strains with different genetic backgrounds in order to facilitate further genetic analysis. The polifungin method (Bal et al., 1974) for the enrichment of auxotrophs after mutagenesis was applied on hypoxanthine-supplemented medium. Only $a d A$ and $a d B$ mutants cannot grow on this medium (see Fig. 1) and hence should be selectively isolated. A total of 559 such mutants were isolated after screening of 41376 colonies (Table 1) and were examined for adenylosuccinate lyase activity and 5-amino-4-imidazole( $N$-succinylo-carboximide)ribotide (SAICAR) accumulation. It was previously observed (Giles et al., 1957; Foley et al., 1965) that only adA mutants accumulate this metabolite, while $a d B$ mutants accumulate hypoxanthine. On the basis of the above tests 25 mutants were assigned as $a d A$. Of these some were obtained from the same polifungin selection and therefore could represent clones of a single original mutant (Bal et al., 1974). Only one mutant from each polifungin selection was used in further tests. 


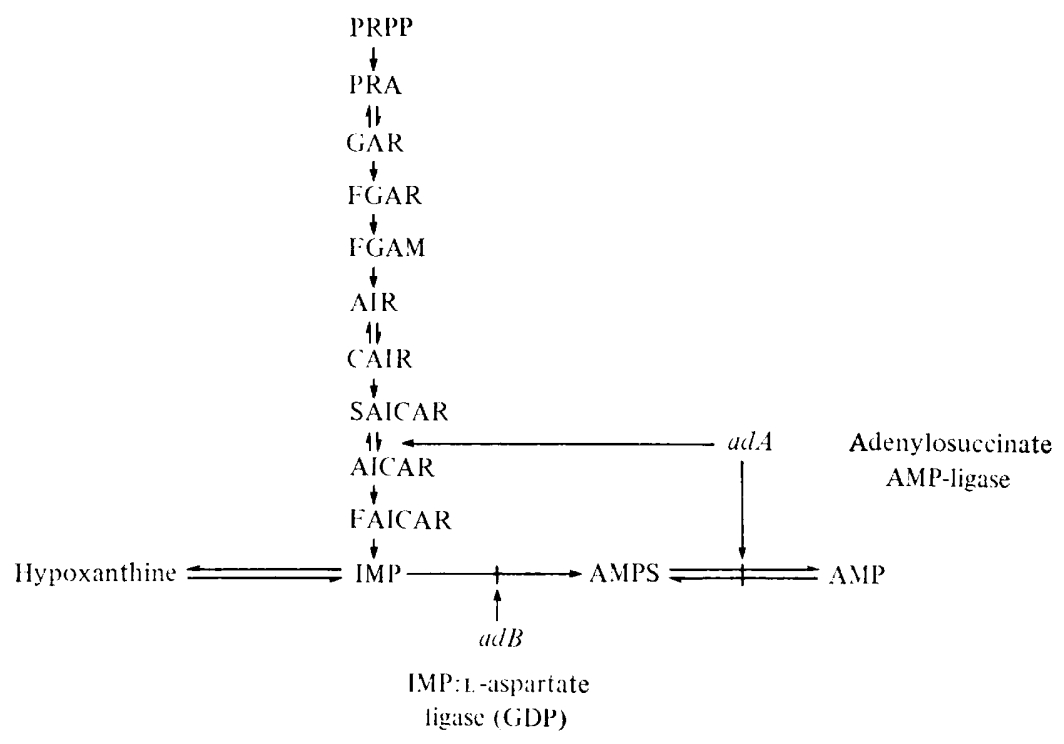

Fig. 1. Pathway of adenine biosynthesis in Aspergillus nidulans (Foley et al., 1965). Positions of metabolic blocks in $a d A$ and $a d B$ mutants are shown. PRPP, 5-Phosphoribosyl-1-pyrophosphate; PRA, phosphoribosylamine; GAR, phosphoribosyl-glycinamide; FGAR, phosphoribosyl-formylglycinamide; FGAM, phosphoribosyl-formylglycinamidine; AIR, phosphoribosyl-aminoimidazole; CAIR, phosphoribosyl-aminoimidazole carboxylate; SAICAR, 5-amino-4-imidazole( $N$-succinylo-carboximide)ribotide; AICAR, phosphoribosyl-aminoimidazole carboxamide; FAICAR, phosphoribosyl-formamido-imidazole carboxamide; IMP, inosine 5'-monophosphate; AMPS, adenylosuccinate; AMP, adenosine 5'-monophosphate.

Table 1. Isolation of mutations in the adA gene following treatment with four mutagens In all cases the polyifungin method for enrichment of auxotrophs was used.

\begin{tabular}{|c|c|c|c|c|c|c|c|}
\hline \multirow[b]{2}{*}{ Mutagen* } & \multirow{2}{*}{$\begin{array}{l}\text { No. of } \\
\text { colonies } \\
\text { tested }\end{array}$} & \multirow{2}{*}{$\begin{array}{l}\text { No. of } \\
\text { experi- } \\
\text { ments }\end{array}$} & \multicolumn{2}{|c|}{$\begin{array}{c}\text { Hypoxanthine } \\
\text { non-utilizing } \\
\text { mutants }\end{array}$} & \multicolumn{2}{|c|}{$a d A$ mutants } & \multirow[b]{2}{*}{ Symbols of adA mutants } \\
\hline & & & No. & $\%$ & No. & $\%$ & \\
\hline U.v. & 17397 & 19 & 117 & 0.67 & 4 & $0 \cdot 023$ & $24,25,101,116$ \\
\hline NQO & 9113 & 14 & 378 & $4 \cdot 1$ & 12 & $0 \cdot 13$ & $\begin{array}{l}1,11,12,15,36,45, \\
48,51,54,115,117,120\end{array}$ \\
\hline MNNG & 9946 & 11 & 56 & 0.56 & 9 & 0.090 & $\begin{array}{l}202,204,210,213,224 \\
226,264,280,289\end{array}$ \\
\hline EMS & 4920 & 9 & 8 & $0 \cdot 16$ & - & - & - \\
\hline Total & 41376 & 53 & 559 & $1 \cdot 35$ & 25 & 0.06 & \\
\hline
\end{tabular}

* U.v., Ultraviolet irradiation; NQO, 4-nitroquinoline-1-oxide; MNNG, $N$-methyl- $N^{\prime}$-nitro- $N$-nitrosoguanidine; EMS, ethyl methanesulphonate.

\section{Analysis of adA mutations}

'Classical' nonsense mutations usually have several properties. They are non-leaky, do not complement with other mutations within the same locus, have a high rate of reversion and their phenotype is not reversed under different growth conditions. The results of tests performed in order to select the adA mutants which best fulfilled the criteria of strains carrying nonsense mutations are given in Tables 2 and 3 and in Fig. 2. The adA24 mutant met all the requirements: it did not complement with any other adA mutant tested (Fig. 2), it reverted with a frequency higher than that of the other $a d A$ mutants (Table 3), and, in all analysed cases, reversion was due to a suppressor mutation (Table 4). The properties of some other adA mutants show that they could carry nonsense mutations too. However, most of the subsequent experiments were performed with the adA24 mutant and its suppressors. 
Table 2. Growth of adA mutants on minimal medium with or without supplements* at two different temperatures

$25^{\circ} \mathrm{C}$

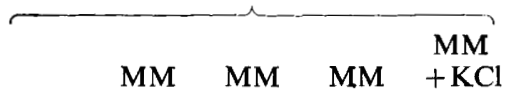

MM $\mathrm{MM}$ MM $\mathrm{MM}+\mathrm{KCl}$

Wild-type

adAl, 11, 12, 15, 24

$36,45,48,51,54$

$101,120,202,213$ adA25

$\begin{array}{ccccc}\mathrm{MM} & +\mathrm{ad} & + \text { hypo } & +\mathrm{KCl} & +\mathrm{ad} \\ + & + & + & + & +\end{array}$

$\begin{array}{lllll}+ & + & + & + & + \\ - & + & - & - & +\end{array}$

$+\quad+\quad+$ $37^{\circ} \mathrm{C}$

MM MM MM + MM

$\mathrm{MM}+\mathrm{ad}$ thypo $+\mathrm{KCl}+\mathrm{ad}$

* $\mathrm{MM}$, minimal medium; ad, adenine $\left(100 \mu \mathrm{g} \mathrm{ml}^{-1}\right)$; hypo, hypoxanthine $\left(75 \mu \mathrm{g} \mathrm{ml}^{-1}\right) ; \mathrm{KCl}\left(150 \mathrm{mg} \mathrm{ml}^{-1}\right)$.

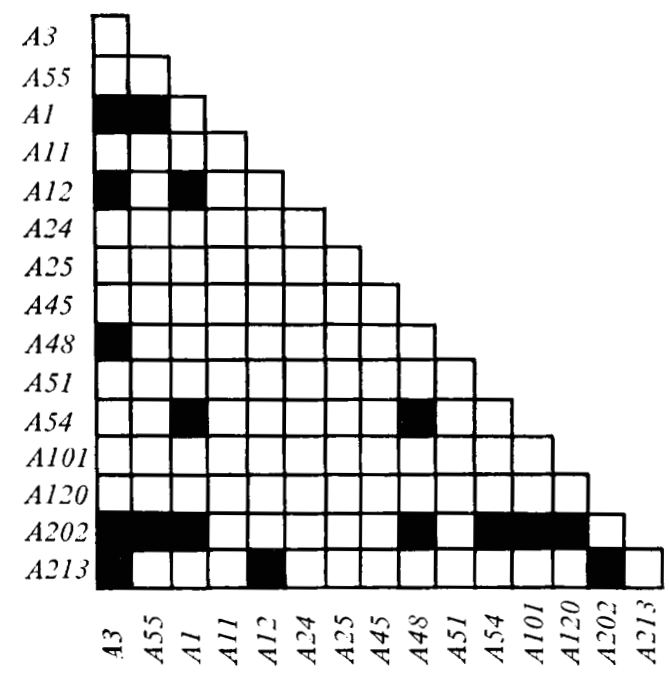

Fig. 2. Results of complementation tests between mutations in the $a d A$ locus: $\mathbf{\square}$, complementation; $\square$, lack of complementation.

\section{Properties and genetic analysis of suppressor mutations}

As mentioned above, reversion of the adA24 mutant was due to suppressor mutations. These suppressors were tested for dominance, and the suppressed strains were tested for their growth properties and adenylosuccinate lyase activity (Table 5). Growth tests with heterokaryons produced between particular suppressed strains and an $a d A 24 s u^{+}$strain showed that all suppressors are dominant. Some of the suppressors were also tested for dominance in diploids and the results of these tests were in full agreement with these obtained in heterokaryons. All suppressed strains grew more slowly than the wild-type on the minimal medium while on adenine-supplemented medium these differences were not observed. This suggests that the slow growth of suppressed strains on minimal medium is due to low efficiency of suppression and is not a property of the suppressor mutation itself. The same conclusion can be drawn from the fact that a strain carrying the $s u-23$ mutation alone (i.e. $a d A^{+} s u-23$ ) grew at the same rate as the wild-type on minimal medium. On the other hand, there was no correlation between the rate of growth on adenine-less medium and the activity of adenylosuccinate lyase of suppressed strains (Table 5). Generally, in suppressed strains the level of adenylosuccinate lyase did not exceed $30 \%$ of wild-type activity. In 14 suppressed strains no activity was detected, probably due to low sensitivity of the assay. 
Table 3. Frequency of reversion of adA mutations

\begin{tabular}{lccc} 
& \multicolumn{3}{c}{ Frequency of reversion* } \\
\cline { 2 - 4 } Mutant symbol & $\begin{array}{c}\text { Spontaneous } \\
\text { NQO-induced }\end{array}$ & MNNG-induced \\
adA1 & $<10^{-12}$ & $<10^{-9}$ & $<10^{-8}$ \\
adA11 & $<10^{-12}$ & $4.0 \times 10^{-9}$ & $4.5 \times 10^{-9}$ \\
adA12 & $1.0 \times 10^{-9}$ & & \\
adA15 & $2.6 \times 10^{-9}$ & & \\
adA24 & $2.8 \times 10^{-6}$ & $6.4 \times 10^{-5}$ & $9.0 \times 10^{-6}$ \\
adA25 & $3.6 \times 10^{-9}$ & & \\
adA36 & $<10^{-12}$ & & \\
adA45 & $2.4 \times 10^{-8}$ & & $5.3 \times 10^{-7}$ \\
adA48 & $4.9 \times 10^{-11}$ & $9.0 \times 10^{-7}$ & \\
adA51 & $3.7 \times 10^{-8}$ & & $3.5 \times 10^{-7}$ \\
adA54 & $8.8 \times 10^{-11}$ & $2.5 \times 10^{-6}$ & $<10^{-8}$ \\
adA101 & $<10^{-12}$ & $<10^{-9}$ & $2.9 \times 10^{-8}$ \\
adA120 & $1.6 \times 10^{-12}$ & $3.2 \times 10^{-7}$ & \\
adA202 & $4.0 \times 10^{-7}$ & & \\
adA213 & $<10^{-13}$ & &
\end{tabular}

* About $10^{10}$ to $10^{12}$ viable conidia were tested in each experiment.

Table 4. Frequency of adenine-requiring colonies in crosses between revertants of the adA24 mutant and the wild-type

\begin{tabular}{|c|c|c|c|c|}
\hline \multirow{2}{*}{$\begin{array}{l}\text { Suppressor } \\
\text { symbol* }\end{array}$} & \multirow[b]{2}{*}{ Origin of revertant } & \multirow{2}{*}{$\begin{array}{l}\text { No. of } \\
\text { colonies } \\
\text { tested }\end{array}$} & \multicolumn{2}{|c|}{$\begin{array}{c}\text { Adenine-requiring } \\
\text { colonies }\end{array}$} \\
\hline & & & No. & $\%$ \\
\hline$s u-22$ & Spontaneous & 126 & 29 & $23 \cdot 0$ \\
\hline$s u-23$ & Spontaneous & 259 & 66 & $25 \cdot 4$ \\
\hline$s u-24$ & Spontaneous & 130 & 9 & $6.9 \dagger$ \\
\hline$s u-26$ & Spontaneous & 70 & 14 & $20 \cdot 0$ \\
\hline$s u-27$ & Spontaneous & 148 & 46 & $31 \cdot 1$ \\
\hline$s u-28$ & Spontaneous & 129 & 31 & $24 \cdot 0$ \\
\hline$s u-29$ & Spontaneous & 78 & 23 & $29 \cdot 5$ \\
\hline$s u-34$ & Spontaneous & 428 & 65 & $15 \cdot 2 \dagger$ \\
\hline$s u-36$ & Spontaneous & 143 & 34 & $23 \cdot 7$ \\
\hline$s u-39$ & Spontaneous & 127 & 42 & $33 \cdot 1$ \\
\hline$s u-44$ & Spontaneous & 693 & 100 & $14 \cdot 4 \dagger$ \\
\hline$s u-51$ & MNNG-induced & 90 & 26 & $28 \cdot 8$ \\
\hline su-52 & MNNG-induced & 104 & 17 & $16 \cdot 4 \dagger$ \\
\hline$s u-53$ & MNNG-induced & 127 & 23 & $18 \cdot 1$ \\
\hline$s u-54$ & Spontaneous & 130 & 34 & $26 \cdot 1$ \\
\hline$s u-55$ & Spontaneous & 52 & 3 & $5 \cdot 8 \dagger$ \\
\hline$s u-56$ & Spontaneous & 128 & 20 & $15 \cdot 6 \dagger$ \\
\hline$s u-57$ & NQO-induced & 174 & 55 & $31 \cdot 6$ \\
\hline$s u-58$ & NQO-induced & 130 & 38 & $29 \cdot 2$ \\
\hline$s u-59$ & MNNG-induced & 128 & 22 & $17 \cdot 2 \dagger$ \\
\hline$s u-60$ & NQO-induced & 104 & 35 & 33.6 \\
\hline$s u-6 I$ & NQO-induced & 103 & 17 & $16 \cdot 5+$ \\
\hline$s u-62$ & MNNG-induced & 129 & 21 & $16 \cdot 3 \dagger$ \\
\hline$s u-63$ & NQO-induced & 233 & 55 & $23 \cdot 6$ \\
\hline$s u-64$ & Spontaneous & 129 & 38 & $29 \cdot 4$ \\
\hline
\end{tabular}


Table 5. Properties of adA24 su strains

Growth on MM with or without supplements

\begin{tabular}{|c|c|c|c|c|c|c|c|}
\hline & & & & & & & \\
\hline & & & $37^{\circ} \mathrm{C}$ & & & & \\
\hline & Rate of & & & $\mathbf{M M}$ & & & adenylo- \\
\hline Strain & $\begin{array}{c}\mathrm{MM} \\
\text { at } 37^{\circ} \mathrm{C}^{*}\end{array}$ & $\begin{array}{l}\text { MM } \\
+ \text { ad }\end{array}$ & $\begin{array}{c}+\mathrm{KCl} \\
(2 \mathrm{M})\end{array}$ & $\begin{array}{c}+\mathrm{KCl} \\
(2 \mathrm{M})\end{array}$ & $\mathbf{M M}$ & $\begin{array}{l}\text { MM } \\
+\mathrm{ad}\end{array}$ & $\begin{array}{c}\text { lyase } \\
\text { activity }\end{array}$ \\
\hline Wild-type & $+++t$ & + & + & + & + & + & 100 \\
\hline $\operatorname{adA24}$ & - & + & - & + & - & + & $<3.0 \ddagger$ \\
\hline$a d A 24 s u-22$ & ++ & + & - & + & + & + & $5 \cdot 0$ \\
\hline$a d A 24 s u-23$ & ++ & + & - & + & + & + & $<3.0 \%$ \\
\hline$a d A 24$ su-24 & $++t$ & + & + & + & + & + & $12 \cdot 0$ \\
\hline$a d A 24 s u-26$ & $t+t$ & + & - & + & + & + & $26 \cdot 0$ \\
\hline adA24 su-27 & + & + & + & + & + & + & $5 \cdot 0$ \\
\hline$a d A 24 s u-28$ & +++ & + & + & + & + & + & $<3.0 \ddagger$ \\
\hline$a d A 24 s u-29$ & + & + & - & + & - & + & $<3.0 \ddagger$ \\
\hline adA24 su-34 & ++ & + & - & + & + & + & $<3.0_{+}^{+}$ \\
\hline$a d A 24 s u-36$ & +++ & + & - & + & + & + & $<3.0^{+}$ \\
\hline adA24su-39 & + & + & - & + & + & + & $25 \cdot 0$ \\
\hline$a d A 24 s u-44$ & ++ & + & + & + & + & + & $<3.0 \ddagger$ \\
\hline$a d A 24 s u-51$ & ++ & + & - & + & + & + & $28 \cdot 0$ \\
\hline$a d A 24 s u-52$ & ++ & + & + & + & + & + & $<3.0 \%$ \\
\hline adA24 su-53 & ++ & + & + & + & + & + & $<3.0 \ddagger$ \\
\hline adA24 su-54 & $+t$ & + & - & + & + & + & $<3.0 \%$ \\
\hline$a d A 24 s u-55$ & ++ & + & - & + & + & + & $10 \cdot 0$ \\
\hline adA24 su-56 & +++ & + & + & + & + & + & $5 \cdot 0$ \\
\hline adA24 su-57 & +++ & + & - & + & + & + & $<3.0_{+}^{+}$ \\
\hline adA24 su-58 & ++ & + & - & + & + & + & $<3 \cdot 0 \ddagger$ \\
\hline adA24su-59 & +++ & + & + & + & + & + & $<3.0 t$ \\
\hline$a d A 24 s u-60$ & + & + & + & + & + & + & $24 \cdot 0$ \\
\hline adA24 su-61 & ++ & + & - & + & + & + & $12 \cdot 0$ \\
\hline$a d A 24 s u-62$ & +++ & + & + & + & + & + & $26 \cdot 0$ \\
\hline adA24 su-63 & ++ & + & + & + & + & + & $<3.0 \%$ \\
\hline$a d A 24 s u-64$ & $+t+$ & + & + & + & + & + & $12 \cdot 0$ \\
\hline
\end{tabular}

$*++++$, Distinct growth after $24 \mathrm{~h} ;+++$, growth after $24 \mathrm{~h}$ but retarded relative to wild-type; ++ , distinct growth after $48 \mathrm{~h} ;+$, distinct growth after $72 \mathrm{~h}$.

$\dagger$ The wild-type strain specific activity (8.1 $\pm 1 \cdot 8$ units) was taken as $100 \%$.

$\ddagger$ Below sensitivity of assay.

Table 6. Linkage of su-51 to phenA in the cross adA24 su-51 $\times$ phenA2

Distribution of phenotypes

Phenotypes

Ad Phen ${ }^{+}$

$\mathrm{Ad}^{+}$Phen

$\mathrm{Ad}^{+}$Phen $^{+}$

Ad Phen

\begin{tabular}{crc} 
Expected* & \multicolumn{2}{c}{ Observed } \\
$\%$ & No. & \multicolumn{1}{c}{$\%$} \\
$12 \cdot 5$ & 8 & 8.0 \\
$37 \cdot 5$ & 34 & 34.0 \\
$37 \cdot 5$ & 42 & 42.0 \\
$12 \cdot 5$ & 16 & 16.0
\end{tabular}

* Assuming su-51 and phen A are unlinked: linkage would produce a deficiency of Ad Phen ${ }^{+}$and $\mathrm{Ad}^{+} \mathrm{Phen}$ phenotypes and an excess of $\mathrm{Ad}^{+} \mathrm{Phen}^{+}$and $\mathrm{Ad}$ Phen phenotypes. 
Table 7. Linkage of su- 63 to lys $B$ in the cross adA24 su-63 $\times$ lysB5

\begin{tabular}{|c|c|c|c|}
\hline \multirow{3}{*}{$\begin{array}{l}\text { Phenotypes } \\
\text { of progeny }\end{array}$} & \multicolumn{3}{|c|}{ Distribution of phenotypes } \\
\hline & \multirow{2}{*}{$\begin{array}{c}\text { Expected* } \\
\%\end{array}$} & \multicolumn{2}{|c|}{ Observed } \\
\hline & & No. & $\%$ \\
\hline Ad Lys ${ }^{+}$ & $12 \cdot 5$ & 9 & $6 \cdot 7$ \\
\hline $\mathrm{Ad}^{+}$Lys & $37 \cdot 5$ & 42 & $32 \cdot 3$ \\
\hline $\mathrm{Ad}^{+} \mathrm{Lys}^{+}$ & $37 \cdot 5$ & 57 & $43 \cdot 8$ \\
\hline Ad Lys & $12 \cdot 5$ & 22 & $17 \cdot 5$ \\
\hline
\end{tabular}

${ }^{*}$ Assuming $s u-63$ and $l y s B$ are unlinked: linkage would produce a deficiency of $\mathrm{Ad} \mathrm{Lys}^{+}$and $\mathrm{Ad}^{+} \mathrm{Lys}$ phenotypes and an excess of $\mathrm{Ad}^{+} \mathrm{Lys}^{+}$and Ad Lys phenotypes.

Table 8. Specificity of the su-23 mutation for different adA alleles

Strains carrying different $a d A$ alleles were crossed to a strain of genotype adA $A^{+} s u-23$.

\begin{tabular}{lcrc} 
& $\begin{array}{c}\text { No. of } \\
\text { colonies } \\
\text { tested }\end{array}$ & \multicolumn{2}{c}{$\begin{array}{c}\text { Adenine-requiring } \\
\text { colonies }\end{array}$} \\
adA allele & 468 & 234 & $50 \cdot 0$ \\
adA11 & 468 & 134 & $28 \cdot 6^{*}$ \\
adA12 & 282 & 81 & $28 \cdot 7^{*}$ \\
adA15 & 156 & 35 & $23 \cdot 2^{*}$ \\
adA25 & 144 & 66 & $44 \cdot 3$ \\
adA36 & 312 & 133 & $42 \cdot 6$ \\
adA48 & 234 & 73 & $31 \cdot 2^{*}$ \\
adA51 & 130 & 26 & $20 \cdot 0^{*}$ \\
adA101 & 154 & 33 & $21 \cdot 4^{*}$ \\
adA116 & 234 & 50 & $21 \cdot 4^{*}$ \\
adA120 & 720 & 351 & $48 \cdot 7$ \\
adA202 & 175 & 86 & $49 \cdot 0$ \\
adA213 & 289 & 76 & $26 \cdot 4^{*}$ \\
adA3 & 468 & 230 & $49 \cdot 1$ \\
adA55 & & &
\end{tabular}

* Mutations whose phenotype is suppressed. The $\mathrm{chi}^{2}$ test indicates that the observed results are in agreement with a $3 \mathrm{Ad}^{+}: 1$ Ad phenotypic distribution; 20 to $30 \%$ of the adenine prototrophs in the progeny of these crosses showed weak growth and presumably represented the adA su class.

Only one suppressed strain, adA24 su-29, was temperature-sensitive. The temperature sensitivity is not the property of the $s u-29$ mutation alone, as the $a d A 24 s u-29$ strain grew normally at $42{ }^{\circ} \mathrm{C}$ on adenine-supplemented medium (Table 5). The growth of 13 suppressed strains was inhibited by $2 \mathrm{M}-\mathrm{KCl}$. Again, this inhibition was only observed on adenine-less medium and not on adenine-supplemented medium (Table 5).

As all suppressor mutations obtained were dominant, it was not possible to establish the number of suppressor loci by complementation tests. Direct recombination analysis was impossible as in no case did we succeed in crossing two suppressor-carrying strains. In this situation the number of suppressor loci was tentatively established by analysis of the linkage of particular suppressor mutations with other loci. Crosses of the type adA su $\times a d A^{+} s u^{+}$had shown that in several cases the number of adenine-requiring progeny was less than $25 \%$ (Table 4), indicating linkage between $a d A$ and $s u$ loci. Suppressors linked to the $a d A$ locus were tentatively assigned to two loci, one 34 map units $(s u-34,44,52,56,59,61,62)$ and the other 12 map units $(s u-24,55)$ from the adA locus. Other crosses showed that $s u-51$ is linked to the phen $A$ locus (chromosome III) by 32 map units (Table 6) and that $s u-63$ is linked to the lys $B$ locus (chromosome V) by 27 map units (Table 7). By haploidization (about 100 haploids were tested in each experiment) three suppressor mutations (su-36, 37 and 64) 
Table 9. Specificity of adA24 suppressors for mutations in other genes

Strains carrying different adA24 suppressors were crossed to strains with tester mutations in unrelated loci and suppression of the tester mutations was examined.

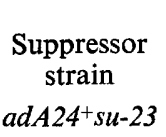

adA $24^{+}$su- 23
Colonies unable to

No. of
colonies

Mutation

examined

lu-2*

lu- $3^{*}$

$l u-4^{*}$

lu-6*

lu-7*

lu-8*

pabaAl

pabaA2

pabaA18

pabaA9

pabaA23

pabaB22

paba-101*

paba-104*

paba-105*

argB2

proAI

proA6

proA7

lys $B 5$

lysF51

nic $A 2$

nic $B 8$

pyroA4

pyro-2*

methE6

methGl

meth $G 2$ *

meth $G 4^{*}$

methG55

methA11*

methA17

methD10

methH2

methH3*

methB41

methB 49

biAl

ino-1*

panto-1*

panto-2*

acr $A 1$

fpaD11

phenA2

riboD5

puA2

fac $A 303$

galA 1

lac AI

adB142*

$a d B I$

adD3

adE20

adF9

adFI7 tested

105

128

129

129

104

130

249

103

146

311

128

283

128

130

129

127

169

144

362

122

257

104

526

171

849

128

182

690

155

71

303

182

194

155

169

147

130

129

517

180

181

182

1562

249

499

867

131

104

102

102

125

128

130

119

122

150 grow on the test medium

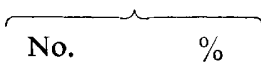

$41 \quad 39 \cdot 0$

$53 \quad 41 \cdot 4$

$55 \quad 42.6$

$70 \quad 53.8$

$41 \quad 39.4$

$58 \quad 44.6$

$121 \quad 48.6$

$45 \quad 43 \cdot 7$

$76 \quad 52.0$

$160 \quad 51 \cdot 4$

$69 \quad 53.9$

$135 \quad 47 \cdot 4$

$55 \quad 43.0$

$72 \quad 55 \cdot 4$

$51 \quad 51.4$

$54 \quad 42 \cdot 5$

$31 \quad 18 \cdot 3 \dagger$

$68 \quad 47 \cdot 2$

$165 \quad 45.6$

$51 \quad 41 \cdot 8$

$113 \quad 44.0$

$45 \quad 39.4$

$244 \quad 46 \cdot 1$

$85 \quad 49 \cdot 7$

$374 \quad 44.0$

$57 \quad 44.5$

$80 \quad 43.9$

$300 \quad 43.5$

$78 \quad 50 \cdot 3$

$35 \quad 49 \cdot 3$

$86 \quad 28 \cdot 1 \dagger$

$84 \quad 46 \cdot 1$

$91 \quad 46.9$

$65 \quad 41 \cdot 9$

$71 \quad 42 \cdot 0$

$32 \quad 21 \cdot 7 \dagger$

$55 \quad 42 \cdot 3$

$57 \quad 44 \cdot 1$

$249 \quad 48 \cdot 2$

$84 \quad 46.6$

$103 \quad 56.9$

$93 \quad 51 \cdot 1$

$742 \quad 47 \cdot 5$

$103 \quad 41 \cdot 4$

$256 \quad 51 \cdot 3$

$439 \quad 50 \cdot 6$

$62 \quad 47 \cdot 3$

$45 \quad 43 \cdot 3$

$52 \quad 51 \cdot 0$

$42 \quad 41 \cdot 2$

$55 \quad 44.0$

$57 \quad 44.5$

$62 \quad 47 \cdot 7$

$54 \quad 45 \cdot 4$

$53 \quad 43 \cdot 4$

$80 \quad 53.3$ 
Table 9 (cont.)

\begin{tabular}{|c|c|c|c|c|}
\hline \multirow{3}{*}{$\begin{array}{l}\text { Suppressor } \\
\text { strain }\end{array}$} & \multirow{2}{*}{$\begin{array}{l}\text { Mutation } \\
\text { examined }\end{array}$} & \multirow{2}{*}{$\begin{array}{l}\text { No. of } \\
\text { colonies } \\
\text { tested }\end{array}$} & \multicolumn{2}{|c|}{$\begin{array}{c}\text { Colonies unable to } \\
\text { grow on the test } \\
\text { medium }\end{array}$} \\
\hline & & & No. & $\%$ \\
\hline & $\begin{array}{l}\text { adG14 } \\
\text { ad } 150 \\
\text { ad-101* } \\
\text { ad-102* }\end{array}$ & $\begin{array}{l}156 \\
130 \\
147 \\
154\end{array}$ & $\begin{array}{l}63 \\
57 \\
64 \\
81\end{array}$ & $\begin{array}{l}40 \cdot 4 \\
43 \cdot 8 \\
43 \cdot 5 \\
52 \cdot 6\end{array}$ \\
\hline$a d A 24 s u-23$ & $a d H 23$ & 171 & 116 & $67 \cdot 8 \ddagger$ \\
\hline $\operatorname{adA24} s u-1 \|$ & $\begin{array}{l}\text { proA10* } \\
\operatorname{lys} B 5\end{array}$ & $\begin{array}{l}389 \\
200\end{array}$ & $\begin{array}{r}93 \\
103\end{array}$ & $\begin{array}{l}23 \cdot 9 \dagger \\
51 \cdot 5\end{array}$ \\
\hline adA24 su-22 & $\begin{array}{l}\text { phen } A 2 \\
\text { lys } B 5 \\
\text { riboD5 }\end{array}$ & $\begin{array}{l}126 \\
126 \\
126\end{array}$ & $\begin{array}{l}57 \\
67 \\
60\end{array}$ & $\begin{array}{l}45 \cdot 2 \\
53 \cdot 2 \\
47 \cdot 6\end{array}$ \\
\hline adA24 su-27 & $a d F 9$ & 148 & 102 & $68 \cdot 9 \ddagger$ \\
\hline adA24su-29 & methG4* & 177 & 80 & $54 \cdot 2$ \\
\hline adA24 su-34 & $\begin{array}{l}\text { riboD5 } \\
\text { lys } B 5 \\
\text { nic } A 2\end{array}$ & $\begin{array}{l}141 \\
405 \\
139\end{array}$ & $\begin{array}{r}58 \\
165 \\
59\end{array}$ & $\begin{array}{l}41 \cdot 4 \\
40 \cdot 7 \\
42 \cdot 4\end{array}$ \\
\hline adA24 su-36 & $\begin{array}{l}\text { lu- } 3^{*} \\
\text { lysB5 } \\
\text { phenA2 } \\
\text { riboD5 }\end{array}$ & $\begin{array}{l}143 \\
143 \\
143 \\
143\end{array}$ & $\begin{array}{l}63 \\
55 \\
58 \\
58\end{array}$ & $\begin{array}{l}44 \cdot 0 \\
38 \cdot 4 \\
40 \cdot 5 \\
40 \cdot 5\end{array}$ \\
\hline$a d A 24 s u-39$ & $\begin{array}{l}\text { methG4* } \\
\text { acr } A 1 \\
\text { phenA2 } \\
\text { lys } B 5\end{array}$ & $\begin{array}{l}148 \\
148 \\
127 \\
127\end{array}$ & $\begin{array}{l}44 \\
65 \\
67 \\
49\end{array}$ & $\begin{array}{l}29 \cdot 7 \dagger \\
43 \cdot 9 \\
52 \cdot 7 \\
38 \cdot 6\end{array}$ \\
\hline$a d A 24 s u-44$ & $\begin{array}{l}\text { adG14 } \\
\text { methG4* } \\
\text { methG2* } \\
\text { phenA2 } \\
\text { lysB5 } \\
\text { paba-106* } \\
\text { riboD5 } \\
\text { methD10 }\end{array}$ & $\begin{array}{l}104 \\
204 \\
129 \\
228 \\
278 \\
278 \\
282 \\
128\end{array}$ & $\begin{array}{r}53 \\
56 \\
72 \\
112 \\
131 \\
125 \\
115 \\
64\end{array}$ & $\begin{array}{l}50 \cdot 9+8 \\
27 \cdot 4 \dagger \\
55 \cdot 8 \\
49 \cdot 1 \\
47 \cdot 1 \\
45 \cdot 0 \\
40 \cdot 8 \\
50 \cdot 0\end{array}$ \\
\hline adA24 su-51 & $\begin{array}{l}\text { lys } B 5 \\
\text { phenA2 } \\
\text { riboD5 }\end{array}$ & $\begin{array}{l}100 \\
100 \\
100\end{array}$ & $\begin{array}{l}57 \\
48 \\
50\end{array}$ & $\begin{array}{l}57 \cdot 0 \\
48 \cdot 0 \\
50 \cdot 0\end{array}$ \\
\hline$a d A 24 s u-52$ & $\begin{array}{l}\text { lys } B 5 \\
\text { phenA2 } \\
\text { riboDS }\end{array}$ & $\begin{array}{l}102 \\
102 \\
102\end{array}$ & $\begin{array}{l}64 \\
62 \\
42\end{array}$ & $\begin{array}{l}62 \cdot 7 \\
60 \cdot 8 \\
41 \cdot 2\end{array}$ \\
\hline adA24 su-54 & $\begin{array}{l}\text { lysB5 } \\
\text { phenA2 } \\
\text { riboDS }\end{array}$ & $\begin{array}{l}127 \\
127 \\
127\end{array}$ & $\begin{array}{l}58 \\
54 \\
54\end{array}$ & $\begin{array}{l}45 \cdot 7 \\
42.5 \\
42 \cdot 5\end{array}$ \\
\hline adA24 su-56 & $\begin{array}{l}\text { lys } B 5 \\
\text { phenA2 } \\
\text { riboD5 }\end{array}$ & $\begin{array}{l}128 \\
128 \\
128\end{array}$ & $\begin{array}{l}57 \\
55 \\
50\end{array}$ & $\begin{array}{l}44 \cdot 5 \\
43 \cdot 0 \\
39 \cdot 0\end{array}$ \\
\hline adA24 su-57 & $\begin{array}{l}\text { pabaAl } \\
\text { riboD5 } \\
\text { tryp } B 403\end{array}$ & $\begin{array}{l}122 \\
122 \\
148\end{array}$ & $\begin{array}{l}68 \\
60 \\
82\end{array}$ & $\begin{array}{l}55 \cdot 8 \\
49 \cdot 2 \\
55 \cdot 4\end{array}$ \\
\hline adA24 su-58 & $\begin{array}{l}\text { lys B5 } \\
\text { phenA2 } \\
\text { riboD5 }\end{array}$ & $\begin{array}{l}129 \\
129 \\
129\end{array}$ & $\begin{array}{l}58 \\
65 \\
71\end{array}$ & $\begin{array}{l}45 \cdot 0 \\
50 \cdot 4 \\
55 \cdot 0\end{array}$ \\
\hline adA24 su-59 & $\begin{array}{l}\text { lys B5 } \\
\text { phen } 22\end{array}$ & $\begin{array}{l}128 \\
128\end{array}$ & $\begin{array}{l}71 \\
70\end{array}$ & $\begin{array}{l}55 \cdot 5 \\
54 \cdot 7\end{array}$ \\
\hline
\end{tabular}


Table 9 (cont.)

\begin{tabular}{|c|c|c|c|c|}
\hline \multirow[b]{2}{*}{$\begin{array}{l}\text { Suppressor } \\
\text { strain }\end{array}$} & \multirow[b]{2}{*}{$\begin{array}{l}\text { Mutation } \\
\text { examined }\end{array}$} & \multirow{2}{*}{$\begin{array}{l}\text { No. of } \\
\text { colonies } \\
\text { tested }\end{array}$} & \multicolumn{2}{|c|}{$\begin{array}{c}\text { Colonies unable to } \\
\text { grow on the test } \\
\text { medium }\end{array}$} \\
\hline & & & No. & $\%$ \\
\hline adA24su-60 & $\begin{array}{l}\text { lys } B 5 \\
\text { phenA2 } \\
\text { riboD5 }\end{array}$ & $\begin{array}{l}103 \\
103 \\
103\end{array}$ & $\begin{array}{l}57 \\
48 \\
50\end{array}$ & $\begin{array}{l}55 \cdot 3 \\
46 \cdot 6 \\
48 \cdot 5\end{array}$ \\
\hline$a d A 24 s u-61$ & lysB5 & 104 & 44 & $42 \cdot 3$ \\
\hline adA24 su-62 & $\begin{array}{l}\text { lys B5 } \\
\text { phenA2 } \\
\text { riboD5 }\end{array}$ & $\begin{array}{l}129 \\
129 \\
129\end{array}$ & $\begin{array}{l}79 \\
62 \\
56\end{array}$ & $\begin{array}{l}61 \cdot 2 \\
48 \cdot 1 \\
43 \cdot 4\end{array}$ \\
\hline adA24 su-63 & $\begin{array}{l}\text { puA2 } \\
\text { lysB5 } \\
\text { lu-14* } \\
\text { phenA2 } \\
\text { riboD5 }\end{array}$ & $\begin{array}{r}103 \\
258 \\
94 \\
104 \\
104\end{array}$ & $\begin{array}{r}49 \\
130 \\
50 \\
49 \\
46\end{array}$ & $\begin{array}{l}47 \cdot 6 \\
50 \cdot 4 \\
53 \cdot 2 \\
47 \cdot 1 \\
44 \cdot 2\end{array}$ \\
\hline adA24 su-64 & $\begin{array}{l}\text { lys } B 5 \\
\text { phenA2 } \\
\text { riboD5 }\end{array}$ & $\begin{array}{l}129 \\
129 \\
129\end{array}$ & $\begin{array}{l}67 \\
57 \\
54\end{array}$ & $\begin{array}{l}52 \cdot 0 \\
44 \cdot 2 \\
42 \cdot 0\end{array}$ \\
\hline
\end{tabular}

* Isolated in the Department of Genetics, Warsaw University.

$\dagger$ Mutations whose phenotype is suppressed by the particular suppressor involved. The chi ${ }^{2}$ test indicates that the observed results are in agreement with a $3 \mathrm{M}^{+}: 1 \mathrm{M}$ phenotypic distribution. Approximately $30 \%$ of the prototrophic progeny in these crosses showed weak growth, and presumably represented the $m s u$ class (where $m / \mathbf{M}$ denotes the mutation/mutant phenotype under test).

$\$$ Theoretically, $37.5 \%$ of adenine-requiring progeny would be expected if the suppressor was effective and $62.5 \%$ if it was not.

$\S$ The $s u-44$ mutation is linked to the adA locus (see Table 4) so the number of adenine-requiring colonies is less than when the mutations tested are unlinked.

|| Further data on the su-1 mutation are given by Bal et al. (1978).

were assigned to linkage group VI. The remaining suppressor mutations do not belong to any of the loci mentioned above. We can therefore assume that the minimum number of adA24 suppressor loci is six.

\section{Determination of suppressor specificity}

The main property of suppressors of nonsense mutations is that they are allele specific and locus non-specific. The specificity of adenine suppressors was studied either by testing the suppressor effect on several different mutations or by selection of simultaneous revertants of two different mutations (one being invariably adA24). The results obtained from the second approach have been presented elsewhere (Bal et al., 1977). Here we present the results obtained by the first approach for the specificity of several adA24 suppressors. Most experiments were carried out with the $s u-23$ mutation.

Specificity of su-23 for adA mutations. The effects of su-23 on 14 different adA mutations were examined. The tests were done in crosses of the type $a d A^{+} s u-23 \times a d A s u^{+}$. As $s u-23$ is unlinked to $a d A, 50 \%$ of adenine-requiring progeny is expected when $s u-23$ is not effective against a given mutation, while only $25 \%$ is expected when the suppressor is effective. On this basis su-23 suppresses 8 out of 14 adA mutations tested (Table 8).

Specificity of adenine suppressors for mutations in lociother than adA. The specificity of the adenine suppressors for mutations in several different loci was examined. Again, the method used was to analyse progeny of crosses between $a d A^{+} s u$ strains and strains carrying the mutations under test. Assuming that the loci of the suppressor and of the mutation tested are not linked, $25 \%$ of progeny showing the mutant phenotype is expected when the suppressor is effective against the test mutation and $50 \%$ when the suppressor is not effective. On 
this basis, 5 out of 62 mutations tested were suppressed by adenine suppressors (Table 9 ). The adenine suppressors $s u-1,23,39,44$ are clearly locus non-specific. Moreover, the results of some crosses strengthen the notion that the suppressors are allele specific. Thus $s u-23$ suppresses methH3 and methG55 but not methG4, methG2, methG1 and methH2; similarly, su-44 suppresses meth $G 4$ but not methG2.

\section{Specificity of suAadE20 and suCadE20 suppressors}

Two adenine suppressor mutations (suAadE20 and $s u C a d E 20$ ) were described by Pritchard (1955). We tested the effect of these suppressors on the adA24 mutation by examining the number of colonies which could not grow on hypoxanthine-supplemented medium among progeny of crosses between suAadE20 adE ${ }^{+}$or $s u C a d E 20$ adE20 strains and the adA24 strain. Of 123 and 534 colonies examined, $44.9 \%$ and $55.3 \%$, respectively, failed to grow on the test medium. This means, that the adA24 mutation is not suppressed by the $s u A$ and $s u C$ mutations.

\section{DISCUSSION}

Aspergillus nidulans serves as a model organism for many kinds of genetical and biochemical studies. Nonsense mutations and their suppressors would be of great use in these studies but have not so far been identified in this organism, although some attempts have been made (H. N. Arst, personal communication). In this paper we describe mutants and suppressors which by genetical criteria can be considered as nonsense mutations and nonsense suppressors. This conclusion is based mainly on the analysis of suppressors of the adA24 mutation. Strains carrying this mutation have all the properties which usually characterize nonsense mutations: non-leakiness, lack of phenotypic reversion under different growth conditions and a high rate of reversion. All revertants of $a d A 24$ strains appeared to carry dominant suppressor mutations, which is expected if we assume, in accordance with the classical scheme of nonsense suppression, that suppressor mutations result in the appearance of a new class of tRNA molecules. That the adenine suppressors are mutations in tRNA genes is further indicated by the mapping of the adA24 suppressors to at least six loci. It is known that tRNA genes in Eukaryota exist in many copies (Schweizer et al., 1969; Dutta \& Ray, 1973; Maleszka, personal communication) and therefore a large number of nonsense suppressor loci is to be expected. Nineteen such loci have been found in Saccharomyces cerevisiae (Hawthorne \& Leupold, 1974) and seven are known both in Schizosaccharomyces pombe (Barben, 1966) and $N$. crassa (Seale, 1972). Thirteen of 25 adenine suppressors examined were sensitive to $\mathrm{KCl}$. This sensitivity may arise from an effect of high osmotic strength (the same results were obtained when $\mathrm{KCl}$ was replaced by glycerol) on the tertiary structure of the mutant tRNA molecule, leading to a reduced efficiency of suppression. Many nonsense suppressors in $S$. cerevisiae produce osmotic-sensitive growth but this sensitivity is a property of the suppressor mutations per se and is not dependent on the presence of a suppressible mutation, as with the present suppressors (Singh, 1977).

The most important property of nonsense suppressors is, however, their allele specificity and locus non-specificity. The adenine suppressors described here have this property as well. Suppressors selected for the adA24 mutation were effective against mutations in four other loci-meth $H$, meth $G$, pro $A$ and $\arg B$. On the other hand, only some alleles in $\operatorname{adA}, \operatorname{meth} G$ and meth $H$ were suppressed while others were not (Table 9).

Suppressors of the $a d A 24$ mutation differ in the extent of growth in the absence of adenine and in the level of adenylosuccinate lyase activity which they restore. However, the activity of the enzyme never exceeded $30 \%$ of the activity in the wild-type. In several cases no adenylosuccinate lyase activity was detected in suppressed strains but we believe that this is due to the insensitivity of the assay procedure. We were not able to detect activities lower than $3 \%$ of that in the wild-type, while it is known that leaky mutants of $N$. crassa, which 
have only $2 \%$ of the wild-type activity of this same enzyme, are still able to grow on adenine-less medium (Giles et al., 1957). The mutation designated $s u-29$ differs from the other suppressor mutations in that it is not effective at $42^{\circ} \mathrm{C}$. This is not unusual for nonsense suppressors. Similar cases were reported in studies on nonsense suppressors in yeast (RasseMessenguy \& Fink, 1973) and Escherichia coli (Gallucci et al., 1970; Oescher \& Woods, 1976). Moreover, in E. coli it was demonstrated that temperature sensitivity was the property of a mutant tRNA molecule (Abelson et al., 1970; Smith et al., 1970). On the other hand, it is possible that in the $a d A 24 s u-29$ strain adenylosuccinate lyase itself becomes temperaturesensitive due to a particular amino acid substitution.

The four locus non-specific suppressors differ in the particular tester mutations they suppress (Table 9). This difference in specificity probably reflects the existence of the three types of nonsense mutations within the sample of mutations tested. The data on suppressor specificity are, however, insufficient to permit speculation on which mutants are amber, ochre or opal type.

Can all mutations suppressed by nonsense suppressors be classified as nonsense mutations? From studies on bacteria and yeasts it is known that from 15 to $30 \%$ of all point mutations in a given gene are nonsense mutations (Singh \& Sherman, 1975), although some exceptions exist (Denis-Duphil \& Lacroute, 1971; Korch \& Snow, 1973). In the adA locus, most thoroughly studied in this work, 8 out of 14 alleles were suppressed by $s u-23$. This apparent high frequency of nonsense mutations is probably due to the method of isolating this series of mutants. All leaky mutants, among which nonsense mutants are not expected, would have been eliminated during polifungin selection. On the other hand, not all mutations which are suppressed by nonsense suppressors can be automatically classified as nonsense mutations. It is known that missense (Newcombe \& Griffiths, 1973; Simarov et al., 1971; Maisurian \& Pozdniakov, 1971; Picard, 1973) and frameshift (Atkins \& Ryce, 1974) mutations can be suppressed by nonsense suppressors. We believe that a similar mechanism of suppression is responsible for the effect of $s u-23$ on the adA101 and adA120 mutants. These two mutants complement with some other mutants with defects in the adA locus and show a very low reversion rate compared to adA24; thus it would be unlikely that these mutants carry nonsense mutations. The same is true for the methH3 mutation; strains carrying this mutation have a much lower reversion rate than those carrying other mutations in the meth $H$ locus (Gajewski \& Litwińska, 1968).

The exceptions mentioned above do not rule out the thesis that the remaining mutations? suppressed by the suppressors of adA24 are nonsense mutations. However, all evidence for this thesis comes from genetical data and should be supported by experiments at the molecular level. Some of these are now in progress.

Locus non-specific suppressors have also been obtained in $A$. nidulans by Roberts $e t$ al . (1978). We have not, however, had an opportunity to test them and to compare them with the suppressors described in this paper.

This work was supported by the Polish Academy of Sciences, within the Project 09.7. We are grateful to Dr Ewa Bartnik for her invaluable help with the manuscript and Mrs Wanda Daczkowska for her excellent technical assistance.

\section{REFERENCES}

Abelson, J. N., Gefter, M. L., LANDy, A., Russell, R. L. \& SмITH, J. D. (1970). Mutant of tyrosine ribonucleic acid. Journal of Molecular Biology 47, 15-28.

Atkins, J. F. \& Ryce, S. (1974). UGA and nontriplet suppressor reading of the genetic code. Nature, London 249, 527-530.

Bal, J. \& PieniążeK, N. J. (1979). Detection of
SAICAR and hypoxanthine accumulation simple method for identification of some purine auxotrophs. Journal of Chromatography 169, 474-476.

Bal, J., Balbin, E. \& Pieniążek, N. J. (1974). Method for isolating auxotrophic mutants in Aspergillus nidulans using $N$-glycosyl-polifungin. Journal of General Microbiology 84, 111-116. 
Bal, J., Bartnik, E., Goryluk, B. \& Pieniążek, N. J. (1975). An easy way of obtaining Aspergillus nidulans haploids in parasexual cycle using $N$-glycosyl-polifungin. Genetical Research 25, 249-252.

Bal, J., Kajtaniak, E. M. \& PieniążeK, N. J. (1977). 4-Nitroquinoline-1-oxide: a good mutagen for Aspergillus nidulans. Mutation Research 56, 153-156.

Bal, J., Maciejko, D. M., Kajtaniak, E. M. \& GAJEWSKI, W. (1978), Supersuppressors in Aspergillus nidulans. Molecular and General Genetics 156, 227-228.

BARBeN, H. (1966). Allelspezifische Suppressor Mutationen von Schizosaccharomyces pombe. Genetica 37, 109-148.

Benzer, S. \& Champe, S. P. (1962). A change from nonsense to sense in the genetic code. Proceedings of the National Academy of Sciences of the United States of America 48, 1114-1121.

Chatroo, B. B. \& Sinha, U. (1974). Mutagenic activity of $N$-methyl- $N^{\prime}$-nitro- $N$-nitrosoguanidine (NTG) and $N$-methyl- $N$-nitrosourea (NMU) in Aspergillus nidulans. Mutation Research 23, 41-49.

Clutterbuck, A. J. (1973). Gene symbols in Aspergillus nidulans. Genetical Research 21, 291-296.

Denis-Duphil, M. \& Lacroute, F. (1971). Fine structure of ura2 locus in Saccharomyces cerevisiae. Molecular and General Genetics 112 , 354-364.

DutTA, S. K. \& RAY, R. (1973). Partial characterization of transfer RNA genes isolated from Neurospora crassa. Molecular and General Genetics 125, 295-300.

FinK, G. R. (1970). The biochemical genetics of yeast. Methods in Enzymology 17A, 59-79.

Foley, J. M., Giles, N. H. \& Roberts, C. F. (1965). Complementation at the adenylosuccinase locus in Aspergillus nidulans. Genetics 52, 1247 1263.

GaJewski, W. \& LiTwińsKa, J. (1968). Methionine loci and their suppressors in Aspergillus nidulans. Molecular and General Genetics 102, 210-220.

Gallucci, E., Pacchetti, G. \& Zangrossi, S. (1970). Genetic studies on temperature sensitive nonsense suppression. Molecular and General Genetics 144, 213-215.

Giles, N. H., Partridge, C. W. \& Nelson, N. J. (1957). The genetic control of adenylosuccinase in Neurospora crassa. Proceedings of the National Academy of Sciences of the United States of America 43, 305-317.

Hawthorne, D. C. \& Leupold, U. (1974). Suppressor mutations in yeast. Current Topics in Microbiology and Immunology 64, 1-47.

HAWTHORNe, D. C. \& MORTIMer, R. K. (1963). Super-suppressors in yeast. Genetics 48, 617-620.

KenNedy, C. \& Crowlesmith, I. (1975). A method for isolating nonsense suppressors in Enterobacteriaceae using a mutant of the drug resistance factor R1. Molecular and General Genetics 138, 359-362.

KoRCH, C. T. \& SNow, R. (1973). Allelic complementation in the first gene for histidine biosynthesis in Saccharomyces cerevisiae. I. Charac- teristics of mutants and genetic mapping of alleles. Genetics 74, 287-305.

Lindsley, D. L. \& Grell, E. H. (1968). Genetic variations of Drosophila melanogaster. Carnegie Institute of Washington, Publication no. 627.

Maisurian, A. N. \& Pozdniakov, V. N. (1971). Suppression of temperature sensitive mutants of bacteriophage $\mathrm{T} 4$ by bacterial suppressors. Molecular and General Genetics 112, 91-92.

Mal.ling, H. V. \& De Serres, F. J. (1970). Genetic effects of $N$-methyl- $N^{\prime}$-nitro- $N$-nitrosoguanidine in Neurospora crassa. Molecular and General Genetics 106, 195-207.

Mindich, L., Cohen, K. \& Weisburd, M. (1976). Isolation of nonsense suppressor mutants in Pseudomonas. Journal of Bacteriology 126, 177-182.

Newcombe, K. D. \& Griffiths, A. J. F. (1973). The suppression of ad-3B mutants by supersuppressors in Neurospora crassa. Genetics 75, 615-622.

Oeschger, M. P. \& Woods, S. L. (1976). A temperature-sensitive suppressor enabling the manipulation of the level of individual proteins in intact cells. Cell 7, 205-212.

PICARD, M. (1973). Genetic evidence for a polycistronic unit of transcription in the complex locus ' 14' in Podospora anserina. II. Genetic analysis of informational suppressors. Genetical Research 21, 1-15.

Piper, P. W., Wasserstein, M., Engbaek, F., Kaltofr, K., Celis, J. E., Zeuthen, J., Liebman, S. \& Sherman, F. (1976). Nonsense suppressors of Saccharomyces cerevisiae can be generated by mutation of the tyrosine tRNA anticodon. Nature, London 262, 757-761.

Pontecorvo, G., Roper, J. A., Hemmons, L. M., MacDonald, K. D. \& Bufton, A. W. J. (1953). The genetics of Aspergillus nidulans. Advances in Genetics 5, 141-238.

Pritchard, R. H. (1955). The linear arrangement of a series of alleles of Aspergillus nidulans. Heredity 9, 343-371.

Randerath, K., Chia, L. S. Y., Gupta, R. C., Randerath, E., Hawkins, E. R., Brum, C. K. \& Chang, S. H. (1975). Structural analysis of nonradioactive RNA by postlabeling: the primary structure of baker's yeast tRNA $\mathrm{CU}_{\mathrm{CUA}}^{\text {leu }}$. Biochemical and Biophysical Research Communications 63, 157-163.

Rasse-Messenguy, F. \& FinK, G. (1973). Temperature-sensitive nonsense suppressors in yeast. Genetics 75, 459-464.

Roberts, T. J., Martinelli, S. D. \& Scazzocchio, C. (1978). Allele-specific suppressor mutations in Aspergillus nidulans. Heredity 41, 129.

Schweizer, H., Mackechnie, C. \& Halvorson, H. O. (1969). The redundancy of ribosomal and transfer RNA genes in Saccharomyces cerevisiae. Journal of Molecular Biology 40, 261-277.

Seale, T. W. (1968). Reversion of the am locus in Neurospora crassa: evidence for nonsense suppression. Genetics 58, 85-99.

SEAlE, T. W. (1972). Super suppressors in Neurospora crassa. I. Induction, genetic localization and relationship to a missense suppressor. Genetics $\mathbf{7 0}$ 385-396. 
Seale, T. W., Brett, M., Baron, A. J. \& Fincham, J. R. S. (1977). Amino acid replacement resulting from suppression and missense reversion of a chain-terminator mutation in Neurospora. Genetics 66, 261-274.

Sherman, F., Stewart, J. W., Parker, J. H., Putterman, G. J., Agrawal, B. B. L. \& MarGoLISH, E. (1970). The relationship of gene structure and protein structure of iso-1-cytochrome $c$ from yeast. Symposia of the Society for Experimental Biology 24, 85-107.

Simarov, B. V., Mironova, L. N. \& Inge-VechTomov, S. G. (1971). Nonsense-missense suppression in yeast. Molecular and General Genetics 113, 302-307.

SiNGH, A. (1977). Nonsense suppressors of yeast cause osmotic-sensitive growth. Proceedings of the National Academy of Sciences of the United Stutes of America 74, 305-309.
Singh, A. \& Sherman, F. (1975). Genetic and physiological characterization of met 15 mutants of Saccharomyces cerevisiae: a selective system for forward and reverse mutations. Genetics 81, 75-97.

Smith, J. D., Barnett, L., BRenNer, S. \& Russell, R. L. (1970). More mutant tyrosine transfer ribonucleic acids. Journal of Molecular Biology $54,1-14$.

Stewart, J. W. \& Sherman, F. (1972). Demonstration of UAG as a nonsense codon in baker's yeast by amino-acid replacements in iso-1-cytochrome $c$. Journal of Molecular Biology 68, 429443.

Stewart, J. W., Sherman, F., Jackson, M., Thomas, F. L. X. \& Shipman, N. (1972). Demonstration of the UAA ochre codon in baker's yeast by amino-acid replacements in iso-1-cytochrome c. Journal of Molecular Biology 68, 83-96. 